\title{
The utility of the aortic dissection team: outcomes and insights after a decade of experience
}

\author{
Nicholas D. Andersen ${ }^{1}$, Ehsan Benrashid ${ }^{1}$, Adia K. Ross ${ }^{2}$, Lisa C. Pickett ${ }^{1}$, Peter K. Smith ${ }^{1}$, Mani A. \\ Daneshmand $^{1}$, Jacob N. Schroder ${ }^{1}$, Jeffrey G. Gaca ${ }^{1}$, G. Chad Hughes ${ }^{1}$ \\ ${ }^{1}$ Department of Surgery, ${ }^{2}$ Department of Medicine, Duke University Medical Center, Durham, NC, USA \\ Correspondence to: G. Chad Hughes, MD, Director. Center for Aortic Disease, Duke University Medical Center, Division of Cardiovascular and \\ Thoracic Surgery, Box 3051, Durham, NC 27710, USA. Email: gchad.hughes@duke.edu.
}

\begin{abstract}
Background: Mortality rates following acute type A aortic dissection (ATAAD) repair are reduced when operations are performed by a high-volume acute aortic dissection (AAD) team, leading to efforts to centralize ATAAD care. Here, we describe our experience with ATAAD repair by our AAD team over the last 10 years, with a focus on patient selection, transfer protocols, operative approach, and volume trends over time.
\end{abstract}

Methods: An AAD team was implemented at our institution in 2005, with dedicated high-volume AAD surgeons, a multidisciplinary approach to thoracic aortic disease management, and a standardized protocol for ATAAD repair. Further process improvements were made in 2013 to facilitate the rapid transfer of ATAAD patients to our institution using stream-lined triage, diagnostic, and transfer protocols for patients with suspected ATAAD (RACE-AD protocol). Volume trends and outcomes were assessed longitudinally over this period.

Results: Institutional ATAAD repair volume remained constant at $12 \pm 2$ cases per year from 2005-2013, but increased nearly two-fold to $22 \pm 6$ cases per year $(\mathrm{P}=0.004)$ from 2013-2015 following implementation of the RACE-AD protocol. To accommodate this increased volume, two additional surgeons were added to the AAD team. Surgeon ATAAD repair volume was unchanged over the 10 -year interval $(7.9 \pm 3.9$ cases per year from 2005-2013 versus 5.5 \pm 1.5 cases per year from 2013-2015; $\mathrm{P}=0.36$ ), and all AAD team surgeons consistently met or exceeded the high-volume surgeon threshold of 5 ATAAD repairs per year. Thirty-day/ in-hospital mortality rates of less than $10 \%$ were maintained over the study period.

Conclusions: Centralization of ATAAD care has begun to occur at our center, with maintenance of low mortality rates for ATAAD repair. These data confirm a net positive impact on regional ATAAD outcomes through transfer of patients to a high-volume center with dedicated AAD surgeons.

Keywords: Aorta; dissection; process improvement

Submitted Mar 29, 2016. Accepted for publication May 23, 2016.

doi: $10.21037 /$ acs.2016.05.12

View this article at: http://dx.doi.org/10.21037/acs.2016.05.12

\section{Introduction}

Volume-outcomes relationships for high-risk surgery are now irrefutable and underlie efforts to centralize complex surgical care (1-3). Acute type A aortic dissection (ATAAD) repair is perhaps the most unpredictable and morbid operation in cardiothoracic surgery, and several studies have shown that mortality rates following ATAAD repair are reduced when operations are performed by high-volume surgeons and high-volume centers (4-6). Although regional networks for the rapid triage and transfer of ST-segment elevation myocardial infarction (STEMI) $(7,8)$ and trauma patients to specialized centers are well established, ATAAD patients are less amenable to centralization than other emergent/unstable patients due to the inability to diagnose ATAAD in the prehospital setting and the exceedingly 
small number of high-volume aortic dissection centers in the United States (4-6). Nonetheless, centralization of acute aortic dissection (AAD) care is an emerging focus of national quality improvement in cardiovascular medicine and likely offers the single best opportunity to improve outcomes from this challenging disease (9-16), aside from prevention (17-19).

Beginning in July of 2005 , we restructured our institutional approach to thoracic aortic disease management based on the hypothesis that performance of operations by a high-volume multidisciplinary aortic surgery team would lead to improved outcomes. The key feature of this program was the performance of nearly all thoracic aortic operations by cardiothoracic surgeons with advanced specialty training in thoracic aortic surgery, including a separate AAD callpool covered only by cardiothoracic surgeons with aortic training. This system led to a modernization of our operative approach to proximal aortic repair and markedly increased surgeon-volume numbers for both elective and non-elective proximal aorta operations. When outcomes of ATAAD repair were specifically assessed six years after program implementation, results showed a dramatic 10-fold reduction in operative mortality compared to the preceding era, despite only a modest increase in institutional ATAAD repair volume. This experience demonstrated that quality improvement measures within a single institution based primarily on increased surgeon specialization were sufficient to normalize outcomes for these challenging cases (12).

Since this report our Thoracic Aortic Surgery Program (TASP) has taken an expanded role in regional ATAAD care through local outreach measures and the development of stream-lined triage, diagnostic, and transfer protocols for patients with suspected or confirmed ATAAD. In this report, we describe our experience with ATAAD repair and the AAD team over the first 10 years of the TASP at Duke University Medical Center, with a focus on patient selection, transfer protocols, operative approach and volume trends over time.

\section{Methods}

\section{Patient population and data collection}

This study was approved by the Institutional Review Board of Duke University and the need for individual patient consent was waived. The multidisciplinary TASP at Duke University Medical Center includes cardiothoracic surgery, vascular surgery, cardiac anesthesia, cardiovascular medicine, cardiac critical care, radiology, neurology, pathology, medical genetics, blood bank, nursing, and perfusion specialists. Elective and non-elective thoracic aortic operations are performed primarily by four principal surgeons who received advanced fellowship training in thoracic aortic surgery. We retrospectively reviewed the records of all patients at our institution diagnosed with spontaneously occurring acute (less than two weeks from symptom onset) type A aortic dissection six years before (June 30, 1999-June 30, 2005) and 10 years after (July 1, 2005December 31, 2015) implementation of the TASP. Patient records were identified from the prospectively-maintained Duke Thoracic Aortic Surgery Database (2005-present) (19) as well as from a query of the Duke Enterprise Data Unified Content Explorer for all patients with a discharge diagnosis code (International Classification of Diseases, Ninth Revision, Clinical Modification, ICD-9CM) of 441.0 (Dissection of aorta) or 441.01 (Dissection of aorta, thoracic). Patients with iatrogenic ATAAD secondary to cardiac surgery or thoracic endovascular aortic repair were excluded from the analysis.

\section{RACE-aortic dissection process flow design and the AAD team}

Beginning in 2013, the RACE-Aortic Dissection (RACEAD) process flow map was implemented at our hospital, in order to improve the efficiency of diagnosis, transfer, and management of patients with AAD. The RACE$\mathrm{AD}$ program was modeled after, and borrows its name from, the Reperfusion of Acute Myocardial Infarction in North Carolina Emergency Departments (RACESTEMI) program, a statewide program implemented in 2006 that outlined prehospital care protocols for the rapid triage and transport of STEMI patients directly to percutaneous coronary intervention (PCI)-capable facilities (7). However, it should be noted that there are no official regulatory policies or hospital agreements in North Carolina which instruct the disposition of acute dissection patients. The RACE-AD program therefore represents an internal process improvement initiative intended to improve the regional outcomes of AAD patients and increase institutional AAD volume through re-allocation of hospital resources and enhanced communication with affiliated and non-affiliated hospitals throughout the Southeast region.

The key features of the RACE-AD transfer protocol 
include immediate communication between the referring physician and the attending aortic surgeon on-call, guided recommendations for prehospital imaging, laboratory, and hemodynamic management, direct patient transfer to the operating room (OR) or cardiothoracic intensive care unit (CTICU), lack of hospital bed divert for any suspected AAD patients, and OR standby within 30 minutes of AAD team activation (Figure 1). The AAD team is led by the aortic attending on-call and activation of the AAD team elicits a batch page to the aortic attending, CT fellow, CT anesthesia attending, CTICU charge nurse, CT OR charge nurse, OR front desk, CTICU attending, and perfusionist on-call, as well as the institutional International Registry of Acute Aortic Dissection (IRAD) coordinator and RACE-AD project manager. Patients requiring emergent operation are transported directly to the OR, bypassing the emergency department and CTICU, and all surgical staff members of the AAD team are expected to have a 30 minute response time. Following completion of an ATAAD repair the aortic attending calls the referring physician to inform them of the results and provide feedback and recommendations for future transfers, thereby strengthening the referral network. A similar RACE-AD process flow map was created to expedite the diagnosis and management of patients presenting directly to our ED with suspected AAD.

\section{Patient selection and operative technique}

Our current approach to ATAAD repair has been previously published (12). Unstable patients (ongoing pain, hemodynamic compromise, tamponade, or malperfusion) and those who present within 48 hours of symptom onset are taken emergently to the operating room for immediate surgical repair. Stable, asymptomatic patients who arrived more than 48 hours after initial symptom onset undergo urgent operation after completion of a preoperative evaluation (20-22). Patients with established visceral malperfusion, especially if static malperfusion or severe limb ischemia with loss of motor function, undergo a "complication-specific approach" in an attempt to first alleviate the malperfusion syndrome $(23,24)$. For patients with debilitating preoperative stroke, operation is delayed by 1-2 weeks in coordination with the neurology service if the patient is hemodynamically stable without rupture or tamponade. Patients with uncomplicated acute type A intramural hematoma with an ascending aortic diameter less than 5 centimeters are managed either medically or surgically (urgent rather than emergent surgery) at the discretion of the attending surgeon (25). Operation is deferred in patients with multi-system organ failure, prohibitive comorbidities, or in patients undergoing cardiopulmonary resuscitation (CPR) with chest compressions. Patients are generally considered unsuitable for transfer if undergoing active CPR or in refractory shock with impending arrest at the outside facility. Further, discussions regarding appropriateness of transfer are held with the referring physician in the case of patients with advanced comorbidities such as severe dementia, nursing home resident and so forth.

All cases are performed by median sternotomy with intraoperative transesophageal echocardiographic and invasive hemodynamic monitoring. Solu-Medrol ( 1 gram, intravenously) is administered for pharmacologic neuroprotection to all patients preoperatively. The standard operation involves aortic valve resuspension with ascending aorta and hemiarch replacement $(26,27)$. Aortic root replacement is performed selectively for aortic root aneurysm, intrinsic aortic valve pathology not amenable to repair, or extensive destruction of the intima of the aortic root by the dissection process. Total arch replacement is similarly performed for aneurysm or extensive destruction of the transverse arch. For hemi- or total arch repair, the open distal anastomosis is completed under a period of moderate hypothermic circulatory arrest (HCA) (28) with intraoperative electroencephalographic (EEG) monitoring, when available $(29,30)$. In the unmonitored cases, patients are cooled until the nasopharyngeal temperature is $<24{ }^{\circ} \mathrm{C}$ as prior work from our group has demonstrated that EEG findings suggestive of cerebral ischemia occur more frequently following onset of HCA at warmer temperatures (28). Adjunctive antegrade cerebral perfusion via the right axillary artery is the preferred cerebral protection strategy in circulatory arrest cases, with retrograde cerebral perfusion being utilized in cases where the right axillary artery is not suitable for cannulation as previously described (26). Transfusion practices are performed as previously described, with intraoperative low-dose recombinant activated factor VII (rFVIIa) and/or prothrombin complex concentrate administered to patients with severe coagulopathy refractory to routine transfusions $(26,31,32)$. Distal arch procedures or attempts to obliterate the descending thoracic aortic false lumen (i.e., frozen elephant trunk procedures) are not routinely performed, but rather, used selectively, such as in the case of a distal arch primary tear (retrograde type A dissection) or markedly 
1. Duke Heart Center

\section{RACE AD \\ Future State Process Flow Map for Patient Transfers}

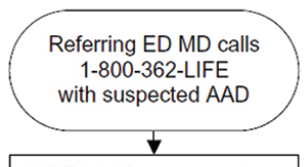

LifeFlight Comms receives call, patches to CT attending on-call and CT ICU Charge Nurse and ED Registration

Aortic CT Attending \& ED MD briefly discuss $(\sim 2 \mathrm{~min})$

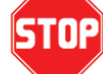

DUH is NEVER on

DIVERT FOR AADs. ? contact the CMO

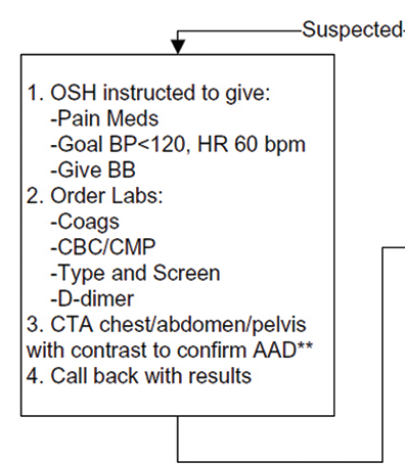

\section{*** Activating the AAD Team}

- Dial 115

- Ask operator "Please page the AAD Hotline team" then state pt

ETA, destination (CTICU or OR), \&

referring facility

- Ex: "AAD Hotline MRN XXXX eta 30

min to OR from outside hospital.

On-call CT Fellow, Anesthesiologist

and Perfusionist please call OR

Front Desk to confirm received page"

Group AAD Hotline team page

CT Fellow On-Call

Aortic CT Attending on-call

CT Anesthesia Attending

CT OR Charge Nurse

OR Front Desk

Perfusion Services

CTICU Charge Nurse

IRAD Coordinator

${ }^{*} \mathrm{CT}$ ICU attending must be notified

by phone. They do not carry pagers.

$$
\text { AAD diagnosis }
$$

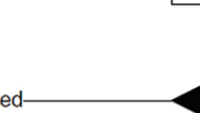

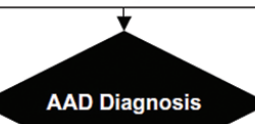

- LifeFlight activates ground or air transport

- LifeFlight activates group page at surgeon request*** - CT ICU CN requests face sheet, EKG, labs, and AAD order set be faxed to the CTICU HUC Station

- ED Registration personnel create MRN/encounter - Armband is created and printed by the CTICU CN and tubed to patient location (OR or CT ICU)

- CT ICU Charge Nurse collects intake info using the

"Acute Thoracic Dissection Intake Form" in Maestro

Care from Referring MD****
Patients must have a confirmed

imaging diagnosis of aortic dissection

performed at the outside hospital in

order for transfer

*** Only LifeFlight or the Aortic CT

Attending or CT Fellow can activate

or cancel the group page

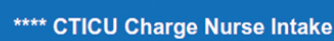
Form available in Maestro Care managed at $\mathrm{OSH}$ or transferred to another facility

\section{If there is no OR} available andlor CTICU is full, LifeFlight will contact the CMO to determine the patient's disposition

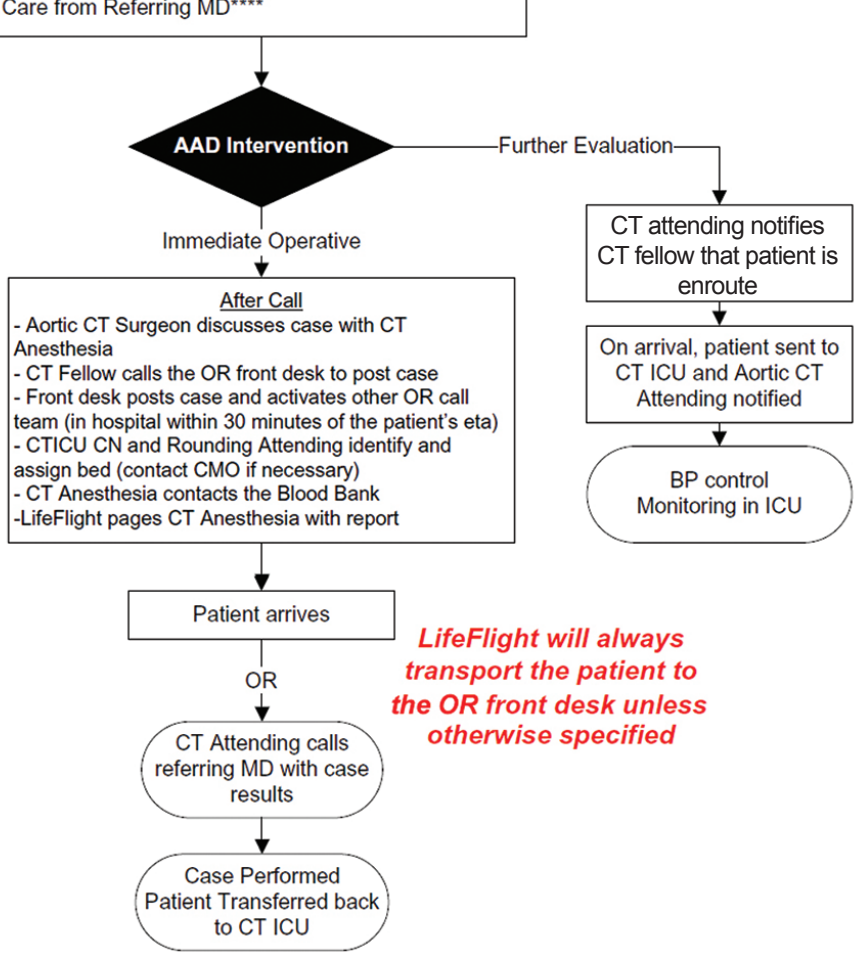

Figure 1 RACE-Aortic Dissection process flow map for patient transfers. 


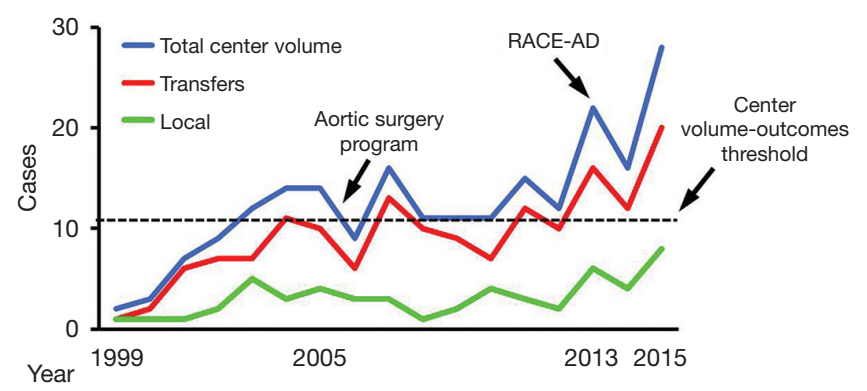

Figure 2 Institutional acute type A aortic dissection volume over a 16-year period. The dashed line denotes the high-volume center threshold associated with superior outcomes in prior studies $(5,6)$.

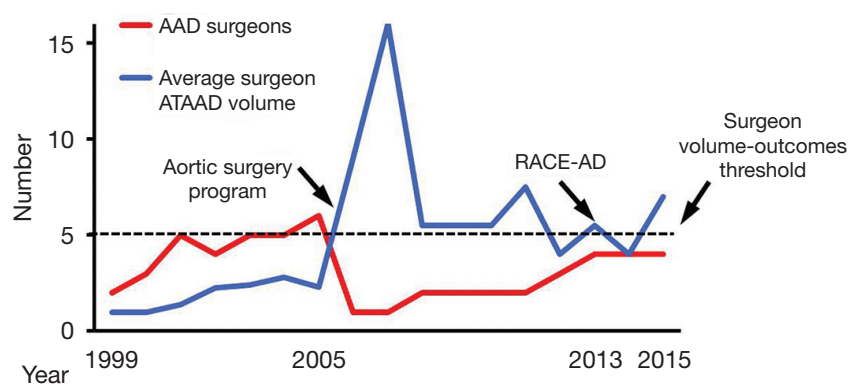

Figure 3 Average surgeon acute type A aortic dissection volume over a 16-year period. The dashed line denotes the high-volume surgeon threshold associated with superior outcomes in prior study (5).

compressed descending aortic true lumen with radiographic malperfusion.

\section{Statistical analysis}

The Fisher's exact test and Student's T test were used for statistical comparisons. Calculations were performed using STATA 11.1 (StataCorp, College Station, TX, USA).

\section{Results}

\section{Institutional ATAAD repair volume}

Previous studies have shown improved outcomes of ATAAD repair at high-volume aortic dissection centers performing more than 11-13 AAD repairs per year (4-6). In the six years prior to implementation of the AAD team (July 1999July 2005), 56 ATAAD repairs were performed yielding an average institutional volume of 9 ATAAD cases per year. Over the first 7.5 years of the AAD team (July 2005December 2012), institutional ATAAD repair volume increased to $12 \pm 2$ ATAAD cases per year (90 cases total), qualifying our program as a high-volume AAD center during this interval. However, ATAAD repair volume nearly doubled after implementation of the RACE-AD transfer protocol in 2013, and averaged $22 \pm 6$ ATAAD cases per year (66 cases total) over the ensuing three calendar years, including 28 ATAAD repairs performed in 2015 alone (Figure 2).

\section{Surgeon ATAAD repair volume}

One prior study identified superior outcomes of ATAAD repair when operations were performed by high-volume $\mathrm{AAD}$ surgeons who performed more than five $\mathrm{AAD}$ repairs per year (5). During the six-year period prior to AAD team implementation, ATAAD operations were performed by 11 different surgeons yielding average annual procedural volumes of approximately two cases per year per surgeon over this period (12). The AAD team began in July of 2005 with one dedicated thoracic aortic surgeon (GCH). In 2008, a second surgeon (JGG) was added and in 2013 two additional surgeons were added (JNS and MAD) in order to accommodate the increasing institutional ATAAD volume associated with the RACE-AD transfer protocol and outreach efforts. The increase in AAD team surgeons closely paralleled the increase in institutional ATAAD repair volume, such that the annual ATAAD repair surgeon-volume remained relatively constant for all participating surgeons at $7.2 \pm 3.5$ ATAAD repairs per year per surgeon $(7.9 \pm 3.9$ cases per year from $2005-2013$ versus $5.5 \pm 1.5$ cases per year from 2013-2015; $\mathrm{P}=0.36$ ), and usually met or exceeded the high-volume surgeon threshold of five ATAAD repairs per year previously associated with superior outcomes (Figure 3) (5).

\section{Operative mortality}

In the six years prior to AAD team implementation, the 30-day/in-hospital operative mortality rate for ATAAD repair was $33.9 \%$. Improved outcomes were consistently maintained over the first 10 years of the AAD team, and the cumulative 30-day/in-hospital operative mortality rate for ATAAD repair was $7.7 \%(\mathrm{P}<0.0001$ vs. the preceding era; Figure 4). Operative mortality rates remained low after implementation of the RACE-AD protocol (5.6\% from 2005-2013 versus 10.6\% from 2013-2015, $\mathrm{P}=0.36$ ). Collectively, these operative mortality rates appeared similar or superior to operative mortality rates reported 


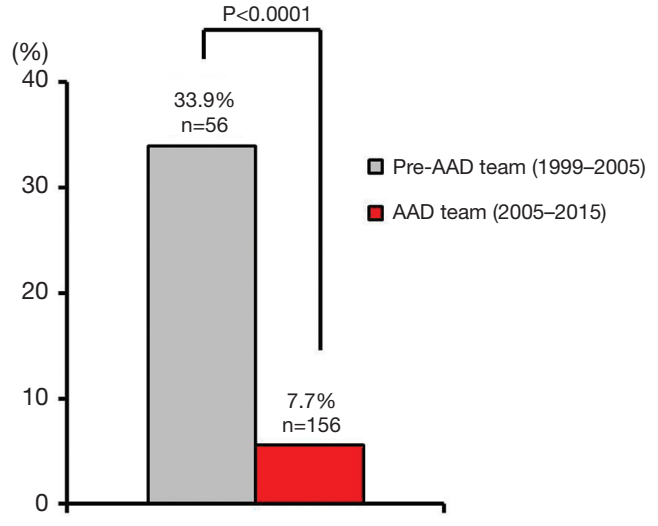

Figure 4 Operative mortality for acute type A aortic dissection repair over a 16-year period. Improved results were sustained over a 10 -year period, with operative mortality rates consistently less than $10 \%$ after AAD team implementation.

nationally from other high-volume AAD centers (12.1$16.4 \%)(5,6)$.

\section{Discussion}

Mounting evidence suggests outcomes of ATAAD repair are improved when operations are performed by high-volume aortic dissection centers and surgeons (4-6,9-16). We aimed to improve our institutional and regional outcomes of ATAAD repair by development of a high-volume AAD team, and here we describe our experience over the first 10 years of program implementation. Results demonstrated that center and surgeon ATAAD repair volumes were consistently maintained at or above the high-volume $\mathrm{AAD}$ repair thresholds associated with superior outcomes (4-6). New transfer protocols and outreach efforts were successful in increasing institutional ATAAD repair volume by nearly two-fold (and rising), and operative mortality rates were consistently maintained below $10 \%$. These data suggest a net positive impact on regional ATAAD outcomes through implementation and promotion of an AAD program, assuming operative mortality rates of $25 \%$ or higher for ATAAD repairs performed at outlying low-volume hospitals (5).

The results from our single-institution experience are consistent with the recent IRAD report demonstrating improved outcomes of ATAAD repair over time associated with an increased proportion of IRAD patients transferred from referring centers (33). These improved national and international outcomes are likely explained partly by era effect and overlapping improvements in infrastructure, surgical technology and techniques, and partly by the expedited diagnosis and transfer of ATAAD patients to highvolume centers with an increased number of operations performed by specialized high-volume AAD surgeons and teams. However, obstacles to the centralization of ATAAD care include the inability to diagnose ATAAD in the prehospital setting, the timeliness of transfer and operation in unstable patients, and the scarcity of high-volume aortic centers in the United States. The latter argument is perhaps the most salient, as studies from the Nationwide Inpatient Sample database suggest only 15-20 centers in the United States meet high-volume criteria and perform more than 11-13 ATAAD repairs per year (4-6). This number is currently about half the number required to allow for one high-volume aortic center for every 5 to 10 million individuals in the United States, or the number predicted to allow most patients diagnosed with ATAAD to reach a referent thoracic aortic center within one to two hours (13). However, our study and others show that regionalization of ATAAD care is possible $(9,10)$, and could likely be expanded through national cooperative efforts, as exemplified by the American Heart Association Lifeline Mission for the rapid diagnosis and transfer of STEMI patients to PCI-capable hospitals $(7,8)$. A similar initiative aimed at concentrating ATAAD patients at one or two designated hospitals in large population centers, as well as improved transport of ATAAD patients to tertiary institutions in rural areas, would produce new high-volume regional aortic receiving centers and presumably improve outcomes (9).

In addition to concentrating ATAAD cases at a small number of centers, our experience also demonstrates the importance of restricting ATAAD cases to a small number of specialized surgeons. Our initial improvement in ATAAD repair outcomes was obtained primarily through an increase in surgeon volume and specialization without a major increase in institutional volume (12). Hence, some large centers that already benefit from a robust volume of dissection cases could further improve outcomes by allocating these cases to a small number of surgeons, as is already done in transplantation and other specialized areas of cardiothoracic surgery $(5,13)$. These initiatives could be implemented immediately within many institutions without relying upon regionalization efforts.

Lastly, the increase in ATAAD volume at our institution was not without growing pains and required considerable buy-in from hospital leadership. The increased number of phone calls, patient evaluations, transfers, and operations required addition of two new surgeons to the AAD 
program and placed an increased burden on ancillary staff and after-hours operating room resources. These volume increases would not have been possible without a significant expansion of our CTICU and OR resources, as well as agreements and negotiations with leadership members to give preference to $\mathrm{AAD}$ patients and to never be 'on divert' for AAD transfers due to a lack of ICU beds. However, with the increased volume of ATAAD cases and expansion of our AAD program we also observed a trend towards increased operative mortality. The explanation for this change could be due to chance (13), surgeon learning curve, a small decrease in surgeon-volume numbers, or a relaxation of patient selection criteria as a result of an increased enthusiasm and willingness to receive and offer surgery to ATAAD patients as a result of our AAD program and transfer protocols. It should also be acknowledged that the small sample size of our single-institution experience limits the utility of statistical comparisons of mortality rates and volume trends between time intervals. Nonetheless, we consider a 30-day/in-hospital ATAAD repair operative mortality rate of $10 \%$ or less to be the current benchmark number for high-volume aortic centers and the general target number for our program (27,34-37).

In summary, creation of an AAD team at our center was associated with improved outcomes of ATAAD repair and the beginnings of centralized ATAAD care in our region. This experience may help to inform the framework and design of other high-volume regional aortic centers.

\section{Acknowledgements}

None.

\section{Footnote}

Conflicts of Interest: The authors have no conflicts of interest to declare.

\section{References}

1. Birkmeyer JD, Siewers AE, Finlayson EV, et al. Hospital volume and surgical mortality in the United States. N Engl J Med 2002;346:1128-37.

2. Birkmeyer JD, Stukel TA, Siewers AE, et al. Surgeon volume and operative mortality in the United States. N Engl J Med 2003;349:2117-27.

3. Finks JF, Osborne NH, Birkmeyer JD. Trends in hospital volume and operative mortality for high-risk surgery. $\mathrm{N}$
Engl J Med 2011;364:2128-37.

4. Knipp BS, Deeb GM, Prager RL, et al. A contemporary analysis of outcomes for operative repair of type A aortic dissection in the United States. Surgery 2007;142:524-8; discussion 528.

5. Chikwe J, Cavallaro P, Itagaki S, et al. National outcomes in acute aortic dissection: influence of surgeon and institutional volume on operative mortality. Ann Thorac Surg 2013;95:1563-9.

6. Iribarne A, Milner R, Merlo AE, et al. Outcomes following emergent open repair for thoracic aortic dissection are improved at higher volume centers. J Card Surg 2015;30:74-9.

7. Fosbol EL, Granger CB, Jollis JG, et al. The impact of a statewide pre-hospital STEMI strategy to bypass hospitals without percutaneous coronary intervention capability on treatment times. Circulation 2013;127:604-12.

8. American Heart Association. Mission: Lifeline Home Page. Available online: http://www.heart.org/HEARTORG/ HealthcareResearch/MissionLifelineHomePage/MissionLifeline-Home-Page_UCM_305495_SubHomePage.jsp

9. Harris KM, Strauss CE, Duval S, et al. Multidisciplinary standardized care for acute aortic dissection: design and initial outcomes of a regional care model. Circ Cardiovasc Qual Outcomes 2010;3:424-30.

10. Davies MG, Younes HK, Harris PW, et al. Outcomes before and after initiation of an acute aortic treatment center. J Vasc Surg 2010;52:1478-85.

11. Graham KJ, Strauss CE, Boland LL, et al. Has the time come for a national cardiovascular emergency care system? Circulation 2012;125:2035-44.

12. Andersen ND, Ganapathi AM, Hanna JM, et al. Outcomes of acute type a dissection repair before and after implementation of a multidisciplinary thoracic aortic surgery program. J Am Coll Cardiol 2014;63:1796-803.

13. Miller DC. Another meiosis in the specialty of cardiovascular and thoracic surgery: birth of the purebred "thoracic aortic surgeon"? J Am Coll Cardiol 2014;63:1804-6.

14. Sales Mda C, Frota Filho JD, Aguzzoli C, et al. Aortic Center: specialized care improves outcomes and decreases mortality. Rev Bras Cir Cardiovasc 2014;29:494-504.

15. Grau JB, Kuschner CE, Ferrari G, et al. Effects of a protocol-based management of type A aortic dissections. J Surg Res 2015;197:265-9.

16. Lenos A, Bougioukakis P, Irimie V, et al. Impact of surgical experience on outcome in surgery of acute type A aortic dissection. Eur J Cardiothorac Surg 2015;48:491-6. 
17. Williams JB, Peterson ED, Zhao Y, et al. Contemporary results for proximal aortic replacement in North America. J Am Coll Cardiol 2012;60:1156-62.

18. Andersen ND, Brennan JM, Zhao Y, et al. Insurance status is associated with acuity of presentation and outcomes for thoracic aortic operations. Circ Cardiovasc Qual Outcomes 2014;7:398-406.

19. Andersen ND, Hanna JM, Ganapathi AM, et al. Insurance status predicts acuity of thoracic aortic operations. J Thorac Cardiovasc Surg 2014;148:2082-6.

20. Davies RR, Coe MP, Mandapati D, et al. Thoracic Surgery Directors Association Award. What is the optimal management of late-presenting survivors of acute type A aortic dissection? Ann Thorac Surg 2007;83:1593-601; discussion 1601-2.

21. Elefteriades JA, Barrett PW, Kopf GS. Litigation in nontraumatic aortic diseases--a tempest in the malpractice maelstrom. Cardiology 2008;109:263-72.

22. Bonser RS, Ranasinghe AM, Loubani M, et al. Evidence, lack of evidence, controversy, and debate in the provision and performance of the surgery of acute type A aortic dissection. J Am Coll Cardiol 2011;58:2455-74.

23. Parsa CJ, McCann RL, Hughes GC. Novel approach to the treatment of distal malperfusion secondary to ascending aortic dissection. J Card Surg 2010;25:220-2.

24. Patel HJ, Williams DM, Dasika NL, et al. Operative delay for peripheral malperfusion syndrome in acute type A aortic dissection: a long-term analysis. J Thorac Cardiovasc Surg 2008;135:1288-95; discussion 1295-6.

25. Kitai T, Kaji S, Yamamuro A, et al. Clinical outcomes of medical therapy and timely operation in initially diagnosed type a aortic intramural hematoma: a 20-year experience. Circulation 2009;120:S292-8.

26. Lima B, Williams JB, Bhattacharya SD, et al. Results of proximal arch replacement using deep hypothermia for circulatory arrest: is moderate hypothermia really justifiable? Am Surg 2011;77:1438-44.

27. Bavaria JE, Pochettino A, Brinster DR, et al. New paradigms and improved results for the surgical treatment of acute type A dissection. Ann Surg 2001;234:336-42; discussion 342-3.

28. Keenan JE, Wang H, Ganapathi AM, et al. Electroencephalography During Hemiarch Replacement With Moderate Hypothermic Circulatory Arrest. Ann Thorac Surg 2016;101:631-7.

29. James ML, Andersen ND, Swaminathan M, et al. Predictors of electrocerebral inactivity with deep hypothermia. J Thorac Cardiovasc Surg 2014;147:1002-7.

30. Stecker MM, Cheung AT, Pochettino A, et al. Deep hypothermic circulatory arrest: I. Effects of cooling on electroencephalogram and evoked potentials. Ann Thorac Surg 2001;71:14-21.

31. Williams JB, Phillips-Bute B, Bhattacharya SD, et al. Predictors of massive transfusion with thoracic aortic procedures involving deep hypothermic circulatory arrest. J Thorac Cardiovasc Surg 2011;141:1283-8.

32. Andersen ND, Bhattacharya SD, Williams JB, et al. Intraoperative use of low-dose recombinant activated factor VII during thoracic aortic operations. Ann Thorac Surg 2012;93:1921-8; discussion 1928-9.

33. Pape LA, Awais M, Woznicki EM, et al. Presentation, Diagnosis, and Outcomes of Acute Aortic Dissection: 17Year Trends From the International Registry of Acute Aortic Dissection. J Am Coll Cardiol 2015;66:350-8.

34. Bekkers JA, Raap GB, Takkenberg JJ, et al. Acute type A aortic dissection: long-term results and reoperations. Eur J Cardiothorac Surg 2013;43:389-96.

35. Girardi LN, Krieger KH, Lee LY, et al. Management strategies for type A dissection complicated by peripheral vascular malperfusion. Ann Thorac Surg 2004;77:1309-14; discussion 1314.

36. Sun L, Qi R, Zhu J, et al. Repair of acute type A dissection: our experiences and results. Ann Thorac Surg 2011;91:1147-52.

37. Westaby S, Saito S, Katsumata T. Acute type A dissection: conservative methods provide consistently low mortality. Ann Thorac Surg 2002;73:707-13.
Cite this article as: Andersen ND, Benrashid E, Ross AK, Pickett LC, Smith PK, Daneshmand MA, Schroder JN, Gaca JG, Hughes GC. The utility of the aortic dissection team: outcomes and insights after a decade of experience. Ann Cardiothorac Surg 2016;5(3):194-201. doi: 10.21037/ acs.2016.05.12 